Supporting Information

\title{
Effect of Metal Coordination Fashion on Oxygen Electrocatalysis of Cobalt-Manganese Oxides
}

\author{
Yuuki Sugawara, ${ }^{* \dagger, \S}$ Hiroaki Kobayashi, ${ }^{*,+, \S}$ Itaru Honma, ${ }^{\dagger}$ and Takeo Yamaguchi ${ }^{*}, \dagger$ \\ ${ }^{\dagger}$ Laboratory for Chemistry and Life Science, Institute of Innovative Research, Tokyo Institute of \\ Technology, R1-17, 4259 Nagatsuta-cho, Midori-ku, Yokohama, Kanagawa 226-8503, Japan \\ ${ }^{\sharp}$ Institute of Multidisciplinary Research for Advanced Materials, Tohoku University, 2-1-1 Katahira, \\ Aoba-ku, Sendai, Miyagi 980-8577, Japan
}

*Corresponding author E-mail: sugawara.y.aa@m.titech.ac.jp; h.kobayashi@tohoku.ac.jp;

yamag@res.titech.ac.jp.

${ }^{\S}$ Y.S. and H.K. contributed equally. 


\section{Supplementary Figures and Tables}
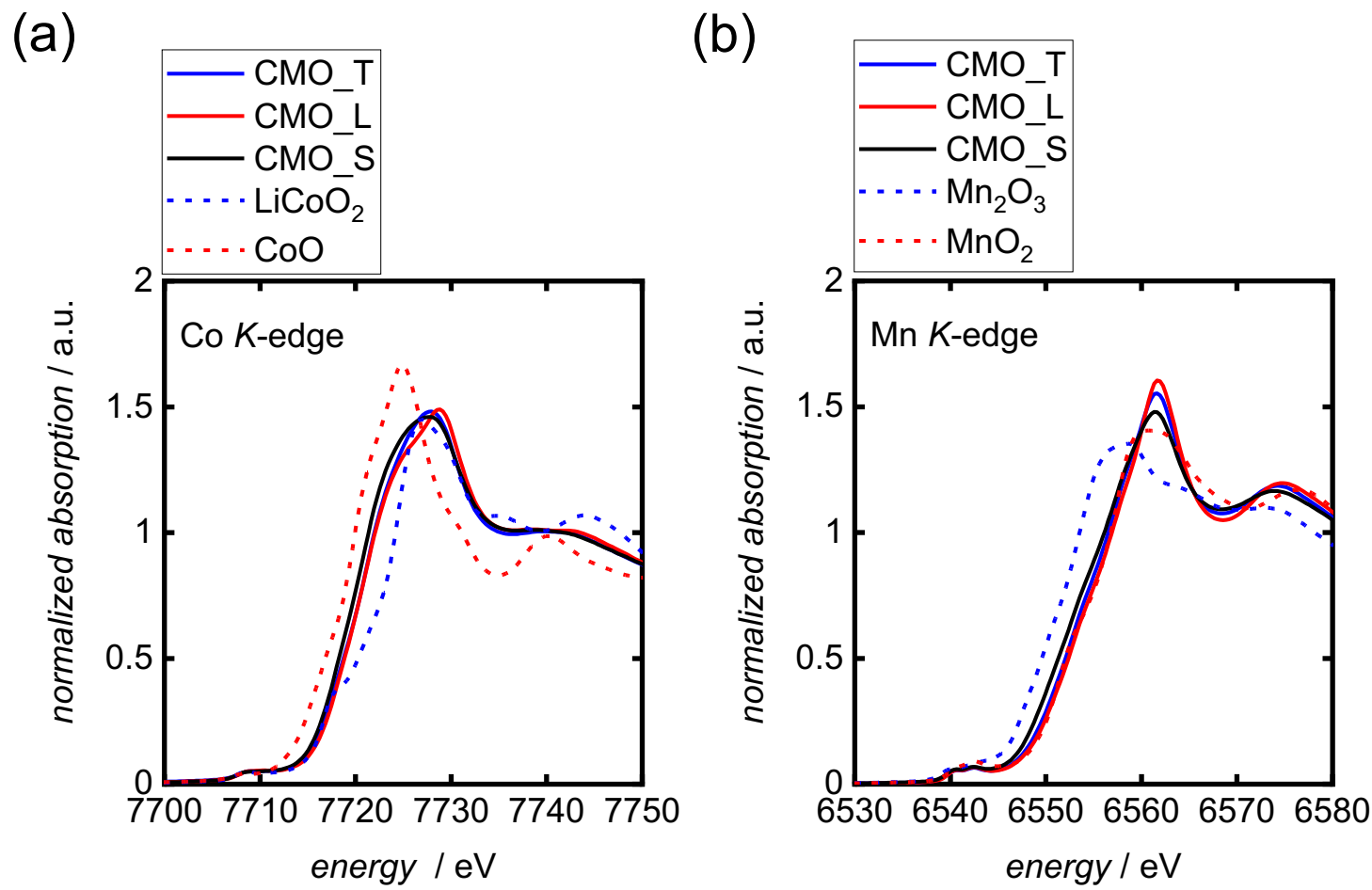

Figure S1. XANES spectra of CMOs. (a) Co and (b) Mn $K$-edge spectra. 
(a)

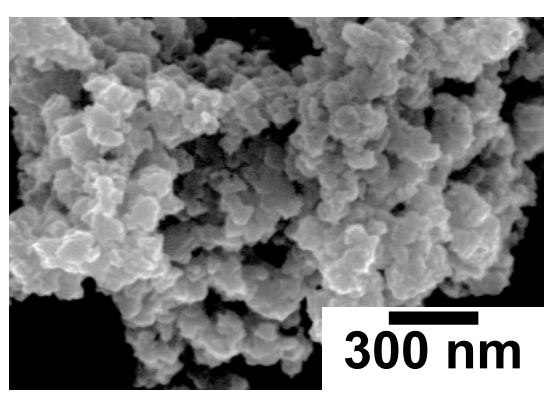

(c)

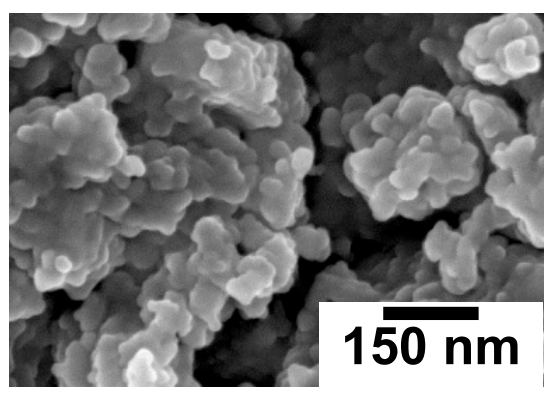

(b)

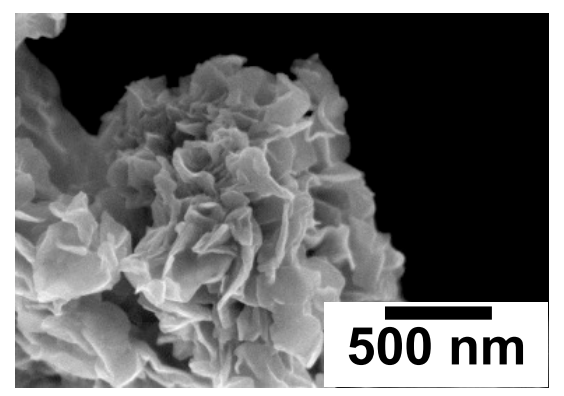

Figure S2. SEM images of (a) $\mathrm{CMO}_{-} \mathrm{T}$, (b) $\mathrm{CMO} L$, and (c) $\mathrm{CMO}$ _S 


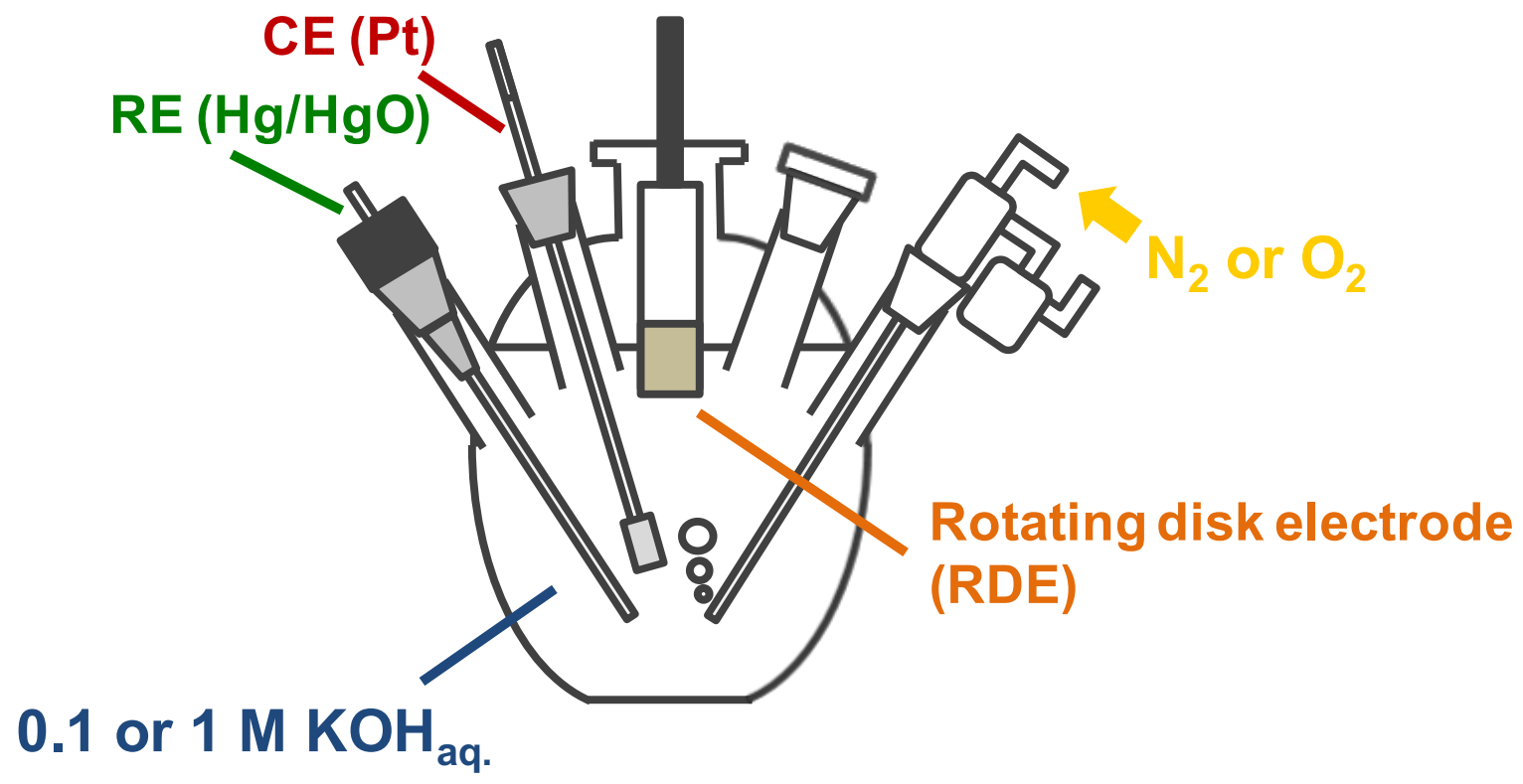

Figure S3. A set-up for electrochemical measurements conducted using RDE. 


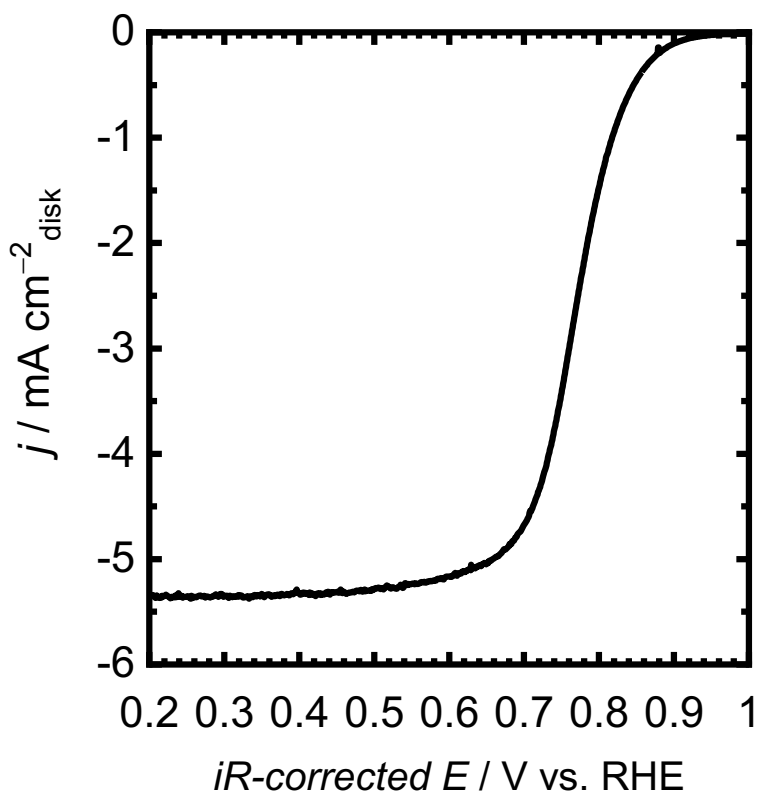

Figure S4. An ORR performance of the commercial Pt/C $(9.8 \% \mathrm{Pt})$. The LSV curve was recorded at an electrode rotating rate of $1600 \mathrm{rpm}$ in $\mathrm{O}_{2}$-saturated $0.1 \mathrm{M} \mathrm{KOH}$. 


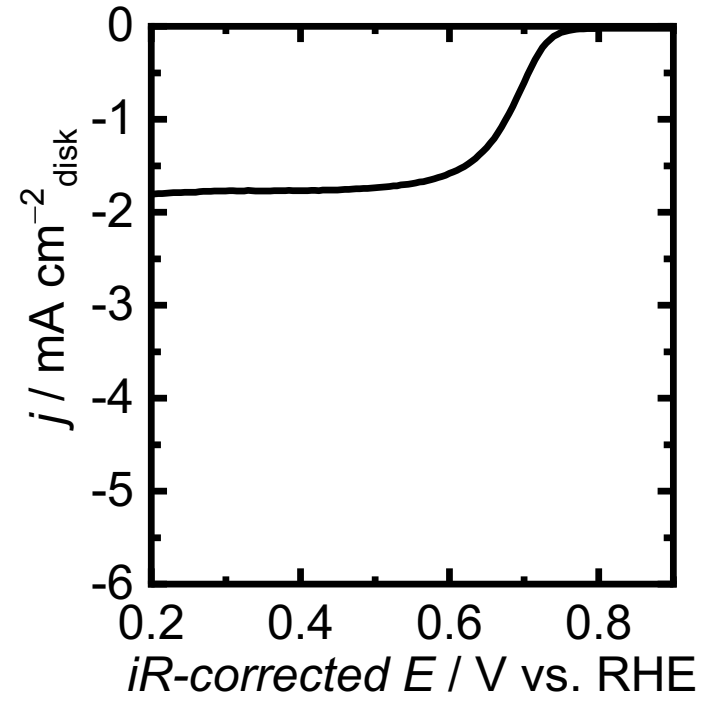

Figure S5. An ORR performance of CNT. The LSV curve was recorded at an electrode rotating rate of $1600 \mathrm{rpm}$ in $\mathrm{O}_{2}$-saturated $0.1 \mathrm{M} \mathrm{KOH}$. 
(a)

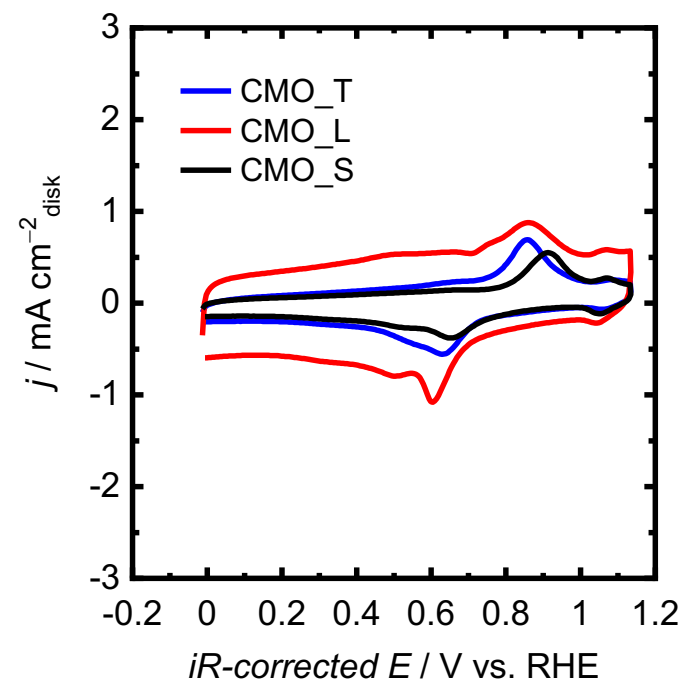

(b)

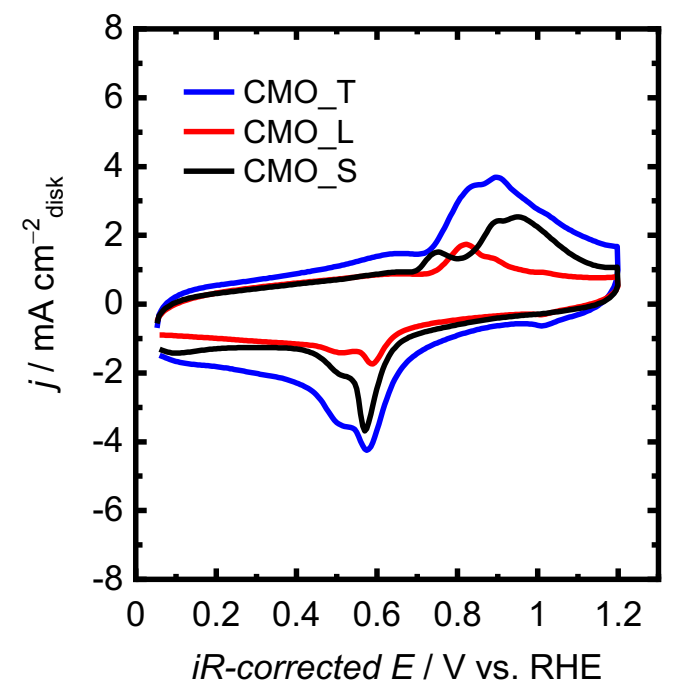

Figure S6. CV curves of CMOs in $\mathrm{N}_{2}$-saturated (a) $0.1 \mathrm{M}$ and (b) $1 \mathrm{M} \mathrm{KOH}$, which were recorded using RDEs for evaluation of ORR and OER activities, respectively. 
(a)

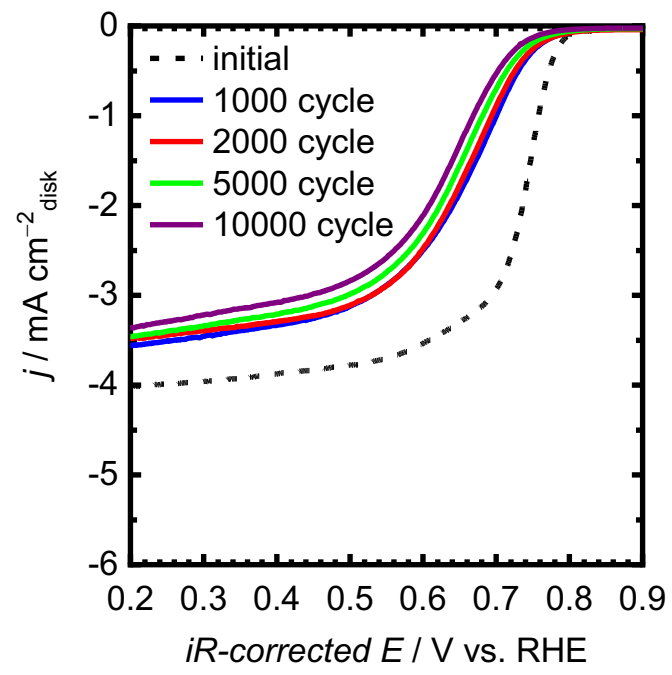

(b)

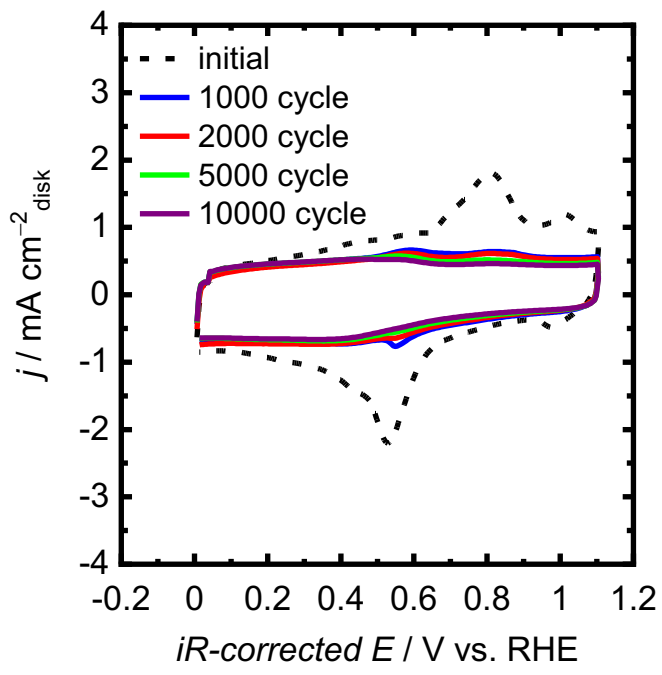

Figure S7. A durability test of ORR performance on CMO_L. (a) LSV curves in $\mathrm{O}_{2}$-saturated $0.1 \mathrm{M} \mathrm{KOH}$ after several load cycles at $60^{\circ} \mathrm{C}$. (b) Change in $\mathrm{CV}$ curves in $\mathrm{N}_{2}$-saturated 0.1 $\mathrm{M} \mathrm{KOH.}$ 


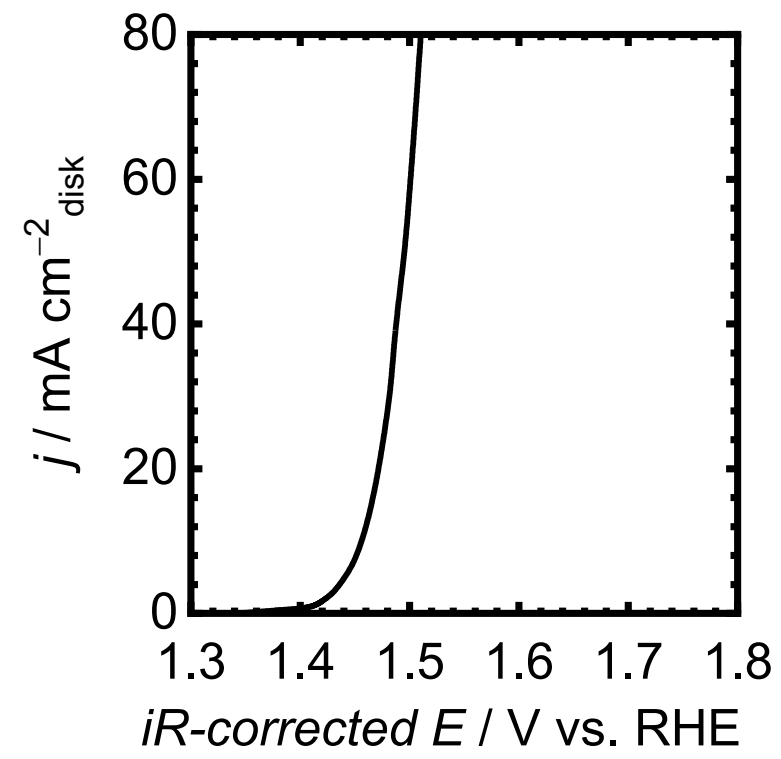

Figure S8. An OER performance of the commercial $\mathrm{IrO}_{2}$. The polarization curve was recorded at an electrode rotating rate of $1600 \mathrm{rpm}$ in $\mathrm{O}_{2}$-saturated $1 \mathrm{M} \mathrm{KOH}$. 
(a)

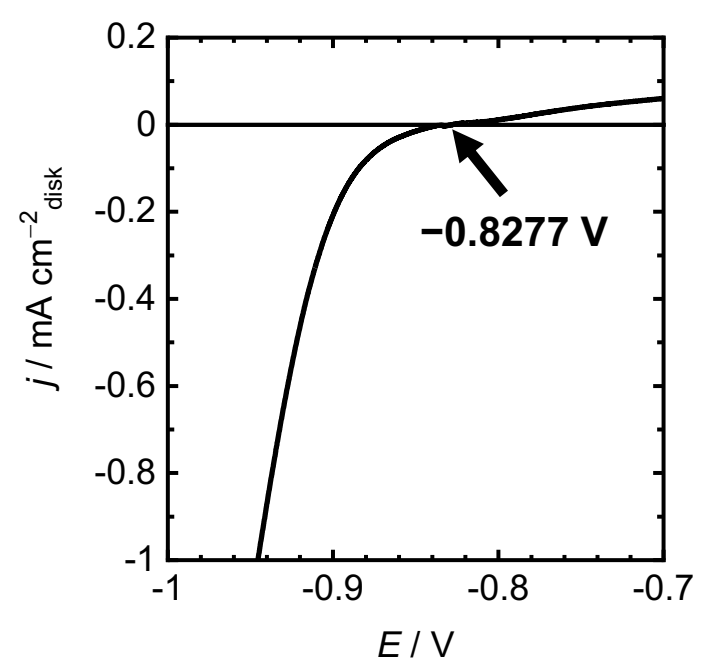

(b)

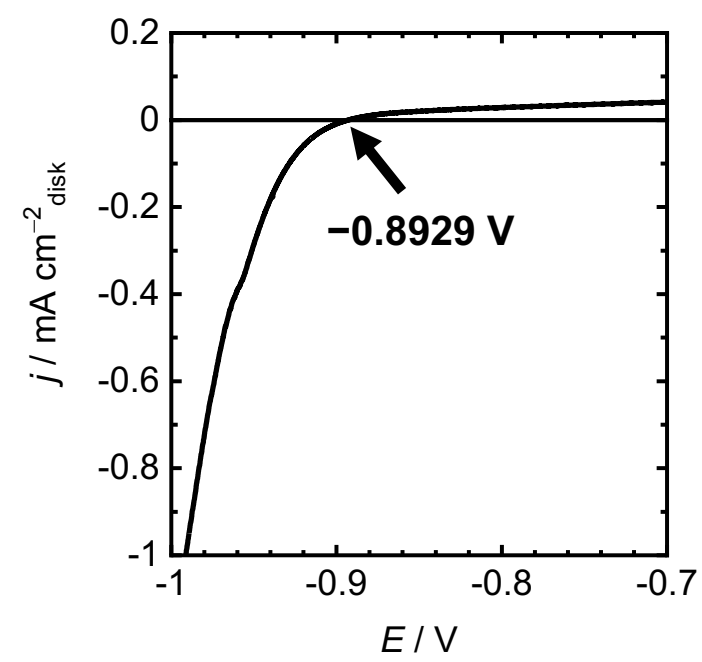

Figure S9. (a) and (b) LSV curves for calibration of the $\mathrm{Hg} / \mathrm{HgO}$ reference electrode for 0.1 and $1 \mathrm{M} \mathrm{KOH}$, respectively. Working and counter electrodes were Pt disc and $\mathrm{Pt}$ wire, respectively. The measurements were performed using $\mathrm{H}_{2}$-saturated $\mathrm{KOH}$ aqueous solutions. 
Table S1. Comparison of ORR and OER activities in alkaline media with previously reported bifunctional metal oxides comprising two $3 d$ transition metals. ${ }^{\text {a }}$

\begin{tabular}{|c|c|c|c|}
\hline $\begin{array}{c}\text { Author and } \\
\text { year }\end{array}$ & Catalyst & $\begin{array}{c}\text { Specific ORR activity @0.75 V } \\
{\left[\mathrm{mA} / \mathrm{cm}^{2}{ }_{\mathrm{BET}}\right]}\end{array}$ & $\begin{array}{c}\text { Specific OER activity @1.65 V } \\
{\left[\mathrm{mA} / \mathrm{cm}^{2}{ }_{\text {BET }}\right]}\end{array}$ \\
\hline Current study & CMO_L & 0.38 & 8.8 \\
\hline Cheng, $2011^{1}$ & $\mathrm{Co}_{1.1} \mathrm{Mn}_{1.9} \mathrm{O}_{4}$ & $2 \times 10^{-2}$ & $2 \times 10^{-2}$ \\
\hline Cheng, $2011^{1}$ & $\mathrm{Co}_{1.28} \mathrm{Mn}_{1.35} \mathrm{O}_{4}$ & $2 \times 10^{-2}$ & $1 \times 10^{-2}$ \\
\hline Yan, $2015^{2}$ & $\mathrm{FeCO}_{2} \mathrm{O}_{4}$ & $8 \times 10^{-4}$ & $3 \times 10^{-3}$ \\
\hline Menezes, $2015^{3}$ & $\mathrm{CoMn}_{2} \mathrm{O}_{4}$ & 0.6 & $4 \times 10^{-2}$ \\
\hline $\mathrm{Li}, 2015^{4}$ & $\mathrm{CoFe}_{2} \mathrm{O}_{4}$ & $5 \times 10^{-3}$ & $4 \times 10^{-2}$ \\
\hline Wang, $2016^{5}$ & $\mathrm{NiCO}_{2} \mathrm{O}_{4}$ & $3 \times 10^{-3}$ & $3 \times 10^{-2}$ \\
\hline $\mathrm{He}, 2016^{6}$ & $\mathrm{NiMn}_{2} \mathrm{O}_{4}$ & $7 \times 10^{-3}$ & $1 \times 10^{-2}$ \\
\hline Wang, $2017^{7}$ & $\mathrm{MnCo}_{2} \mathrm{O}_{4}$ & $9 \times 10^{-2}$ & 0.3 \\
\hline Bin, $2017^{8}$ & $\mathrm{MnCo}_{2} \mathrm{O}_{4}$ & $4 \times 10^{-3}$ & $6 \times 10^{-3}$ \\
\hline $\mathrm{Si}, 2017^{9}$ & $\mathrm{MnFe}_{2} \mathrm{O}_{4}$ & $6 \times 10^{-3}$ & $4 \times 10^{-3}$ \\
\hline $\mathrm{Si}, 2017^{9}$ & $\mathrm{CoFe}_{2} \mathrm{O}_{4}$ & $2 \times 10^{-2}$ & 0.2 \\
\hline $\mathrm{Si}, 2017^{9}$ & $\mathrm{NiFe}_{2} \mathrm{O}_{4}$ & $7 \times 10^{-3}$ & 0.1 \\
\hline Bejar, $2019^{10}$ & $\mathrm{NiCO}_{2} \mathrm{O}_{4}$ & 0.2 & 0.9 \\
\hline
\end{tabular}

${ }^{\mathrm{a}}$ The highlighted catalysts were recorded based on bare metal oxides physically mixed with conductive carbons and excluding chemically composited metal oxide-carbon hybrid materials. 
Table S2. TOF of ORR and OER on CMOs.

\begin{tabular}{|c|c|c|}
\hline \multirow{2}{*}{ Catalyst } & \multicolumn{2}{|c|}{$\mathrm{TOF}\left[\mathrm{s}^{-1}\right]$} \\
\hline & ORR@0.75 V & OER@1.65 V \\
\hline CMO_T & $2.8 \times 10^{-2}$ & 0.37 \\
\hline CMO_L & $2.0 \times 10^{-2}$ & 0.45 \\
\hline $\mathrm{CMO} \mathrm{S}$ & $2.8 \times 10^{-2}$ & 0.14 \\
\hline
\end{tabular}


Table S3. Results of fitting analyses for Co and Mn $K$-edge EXAFS.

\begin{tabular}{|c|c|c|c|c|c|c|c|}
\hline \multirow[b]{2}{*}{ Catalyst } & \multicolumn{3}{|c|}{ First coordination of $\mathrm{Co}-\mathrm{O}$} & \multicolumn{3}{|c|}{ Second coordination of $\mathrm{Co}-\mathrm{M}^{\mathrm{a}}$} & \multirow[b]{2}{*}{ R-factor } \\
\hline & $\begin{array}{c}\text { Coordination } \\
\text { number }\end{array}$ & $\begin{array}{c}\text { Distance } \\
\text { / } \AA\end{array}$ & $\begin{array}{c}\text { Debye-Waller } \\
\text { factor / } \AA^{2}\end{array}$ & $\begin{array}{c}\text { Coordination } \\
\text { number }\end{array}$ & $\begin{array}{c}\text { Distance } \\
/ \AA\end{array}$ & $\begin{array}{c}\text { Debye-Waller } \\
\text { factor / } \AA^{2}\end{array}$ & \\
\hline $\mathrm{CMO}_{-} \mathrm{T}$ & $4.4(11)$ & $1.916(17)$ & $0.009(4)$ & $1.6(5)$ & $2.805(15)$ & $0.002(2)$ & 0.056 \\
\hline CMO_L & $3.7(4)$ & $1.917(8)$ & $0.0051(16)$ & $3.6(4)$ & $2.824(6)$ & $0.0039(9)$ & 0.013 \\
\hline CMO_S & $4.0(9)$ & $1.922(16)$ & $0.008(3)$ & $2.0(6)$ & $2.814(14)$ & $0.003(2)$ & 0.054 \\
\hline \multirow[b]{2}{*}{ Catalyst } & \multicolumn{3}{|c|}{ First coordination of $\mathrm{Mn}-\mathrm{O}$} & \multicolumn{3}{|c|}{ Second coordination of $\mathrm{Mn}-\mathrm{M}^{\mathrm{b}}$} & \\
\hline & $\begin{array}{c}\text { Coordination } \\
\text { number }\end{array}$ & $\begin{array}{c}\text { Distance } \\
/ \AA\end{array}$ & $\begin{array}{c}\text { Debye-Waller } \\
\text { factor / } \AA^{2}\end{array}$ & $\begin{array}{l}\text { Coordination } \\
\text { number }\end{array}$ & $\begin{array}{c}\text { Distance } \\
\text { / } \AA\end{array}$ & $\begin{array}{c}\text { Debye-Waller } \\
\text { factor / } \AA^{2}\end{array}$ & R-factor \\
\hline $\mathrm{CMO}_{-} \mathrm{T}$ & $4.2(5)$ & $1.890(8)$ & $0.0030(13)$ & $3.4(5)$ & $2.851(8)$ & $0.0030(11)$ & 0.018 \\
\hline CMO_L & $4.4(6)$ & $1.890(9)$ & $0.0030(15)$ & $4.2(6)$ & $2.850(8)$ & $0.0030(11)$ & 0.020 \\
\hline CMO_S & $3.7(4)$ & $1.886(8)$ & $0.0027(13)$ & $2.7(5)$ & $2.857(10)$ & $0.0037(15)$ & 0.022 \\
\hline
\end{tabular}

${ }^{\mathrm{a}}$ Calculated using the Co-Mn path. ${ }^{\mathrm{b}}$ Calculated using the $\mathrm{Mn}-\mathrm{Mn}$ path. 


\section{References}

(1) Cheng, F. Y.; Shen, J. A.; Peng, B.; Pan, Y. D.; Tao, Z. L.; Chen, J., Rapid room-temperature synthesis of nanocrystalline spinels as oxygen reduction and evolution electrocatalysts. Nat. Chem. 2011, 3, 7984.

(2) Yan, W. N.; Yang, Z. R.; Bian, W. Y.; Yang, R. Z., FeCo $\mathrm{F}_{4} /$ hollow graphene spheres hybrid with enhanced electrocatalytic activities for oxygen reduction and oxygen evolution reaction. Carbon $\mathbf{2 0 1 5 ,}$ $92,74-83$.

(3) Menezes, P. W.; Indra, A.; Sahraie, N. R.; Bergmann, A.; Strasser, P.; Driess, M., Cobalt-ManganeseBased Spinels as Multifunctional Materials that Unify Catalytic Water Oxidation and Oxygen Reduction Reactions. ChemSusChem 2015, 8, 164-171.

(4) Li, P. X.; Ma, R. G.; Zhou, Y.; Chen, Y. F.; Zhou, Z. Z.; Liu, G. H.; Liu, Q.; Peng, G. H.; Liang, Z. H.; Wang, J., In situ growth of spinel $\mathrm{CoFe}_{2} \mathrm{O}_{4}$ nanoparticles on rod-like ordered mesoporous carbon for bifunctional electrocatalysis of both oxygen reduction and oxygen evolution. J. Mater. Chem. A 2015, $3,15598-15606$.

(5) Wang, J.; Wu, Z. X.; Han, L. L.; Lin, R. Q.; Xin, H. L. L.; Wang, D. L., Hollow-Structured CarbonSupported Nickel Cobaltite Nanoparticles as an Efficient Bifunctional Electrocatalyst for the Oxygen Reduction and Evolution Reactions. ChemCatChem 2016, 8, 736-742.

(6) He, X. B.; Yin, F. X.; Li, Y. H.; Wang, H.; Chen, J. N.; Wang, Y. H.; Chen, B. H., $\mathrm{NiMnO}_{3} / \mathrm{NiMn}_{2} \mathrm{O}_{4}$ Oxides Synthesized via the Aid of Pollen: Ilmenite/Spinel Hybrid Nanoparticles for Highly Efficient Bifunctional Oxygen Electrocatalysis. ACS Appl. Mater. Interfaces 2016, 8, 26740-26757.

(7) Wang, W. H.; Kuai, L.; Cao, W.; Huttula, M.; Ollikkala, S.; Ahopelto, T.; Honkanen, A. P.; Huotari, S.; Yu, M. K.; Geng, B. Y., Mass-Production of Mesoporous $\mathrm{MnCo}_{2} \mathrm{O}_{4}$ Spinels with Manganese(IV)- and 
Cobalt(II)-Rich Surfaces for Superior Bifunctional Oxygen Electrocatalysis. Angew. Chem. Int. Ed. 2017, 56, 14977-14981.

(8) Bin, D.; Guo, Z. Y.; Tamirat, A. G.; Ma, Y. Y.; Wang, Y. G.; Xia, Y. Y., Crab-shell induced synthesis of ordered macroporous carbon nanofiber arrays coupled with $\mathrm{MnCo}_{2} \mathrm{O}_{4}$ nanoparticles as bifunctional oxygen catalysts for rechargeable Zn-air batteries. Nanoscale 2017, 9, 11148-11157.

(9) Si, C. H.; Zhang, Y. L.; Zhang, C. Q.; Gao, H.; Ma, W. S.; Lv, L. F.; Zhang, Z. H., Mesoporous nanostructured spinel-type $\mathrm{MFe}_{2} \mathrm{O}_{4}(\mathrm{M}=\mathrm{Co}, \mathrm{Mn}, \mathrm{Ni})$ oxides as efficient bi-functional electrocatalysts towards oxygen reduction and oxygen evolution. Electrochim. Acta 2017, 245, 829-838.

(10) Bejar, J.; Alvarez-Contreras, L.; Ledesma-Garcia, J.; Arjona, N.; Arriaga, L. G., Electrocatalytic evaluation of $\mathrm{Co}_{3} \mathrm{O}_{4}$ and $\mathrm{NiCo}_{2} \mathrm{O}_{4}$ rosettes-like hierarchical spinel as bifunctional materials for oxygen evolution (OER) and reduction (ORR) reactions in alkaline media. J. Electroanal. Chem. 2019, 847, 113190. 